\title{
Alpha-Fetoprotein Ratio Predicts Alpha-Fetoprotein Positive Hepatocellular Cancer Patient Prognosis after Hepatectomy
}

\author{
Li-Yue Sun $\mathbb{D}^{1}{ }^{1}$ Wen-Jian Cen $\mathbb{D},{ }^{2,3,4}$ Wen-Ting Tang $\mathbb{D}^{2,3,4}$ Ling Deng $\mathbb{D}^{2,3,4}$ \\ Fang Wang $\mathbb{D}^{2,3,4}$ Xiao-Meng Ji $\mathbb{C}^{2,3,4}$ Jiao-Jiao Yang $\mathbb{D}^{2,3,4}$ Ren-Jing Zhang $\mathbb{D}^{2,3,4}$ \\ Xu-Hui Zhang $\mathbb{D}^{1}{ }^{1}$ and Zi-Ming Du $\mathbb{D}^{2,3,4}$ \\ ${ }^{1}$ Second Department of Oncology, Guangdong Second Provincial General Hospital, 466 Xingang-Zhong Road, Guangzhou, China \\ ${ }^{2}$ State Key Laboratory of Oncology in South China, Guangzhou, China \\ ${ }^{3}$ Collaborative Innovation Center for Cancer Medicine, Guangzhou, China \\ ${ }^{4}$ Department of Molecular Diagnostics, Sun Yat-sen University Cancer Center, Guangzhou, China
}

Correspondence should be addressed to Xu-Hui Zhang; 9158863@qq.com and Zi-Ming Du; duzm1@sysucc.org.cn

Received 9 October 2021; Accepted 22 December 2021; Published 11 January 2022

Academic Editor: Jie Mei

Copyright (c) 2022 Li-Yue Sun et al. This is an open access article distributed under the Creative Commons Attribution License, which permits unrestricted use, distribution, and reproduction in any medium, provided the original work is properly cited.

\begin{abstract}
Background. This study was conducted to investigate the effect of alpha-fetoprotein (AFP) ratio on the prognosis of AFPpositive hepatocellular carcinoma (HCC) patients after hepatectomy. Methods. We retrospectively included 879 HCC patients with AFP-positive who underwent hepatectomy from February 2012 to October 2017 and randomly divided into training cohort and validation cohort. AFP ratio was equal to the AFP level within one week before hepatectomy to AFP level within 20-40 days after surgery. The end point of follow-up was disease-free survival (DFS) and overall survival (OS). Results. AFP ratio was not associated with clinical characteristics in training cohort and validation cohort. According to the X-tile software, the optimum cut-off point was 17.8 for AFP ratio. Significant differences between AFP ratio high and AFP ratio low were observed in DFS and OS in both cohort $(p<0.05)$. Kaplan-Meier curves and receiver-operating curves were showed that AFP ratio was better than AFP level preoperation in predicting the prognosis of AFP-positive HCC patients after hepatectomy. The multivariate analysis demonstrated that AFP ratio was a significant independent risk factor for both OS and DFS in HCC patients with AFP-positive. Conclusions. AFP ratio might be a prognosis predictor for HCC patients with AFP-positive after hepatectomy.
\end{abstract}

\section{Introduction}

Hepatocellular carcinoma (HCC) is a common malignancy of the digestive system in China [1]. Hepatectomy remains the most effective treatment for HCC patient without metastasis [2]. However, there existed conventional prognostic indicators that are poor at predicting the prognosis of HCC after hepatectomy [3]. Alpha-fetoprotein (AFP) has been used as an indicator of HCC diagnosis and prognosis, but only $60 \%$ of patients with HCC have positive AFP [4]. Previous studies have shown that AFP-positive was associated with worse biological behavior and inferior survival compared with AFP-negative patients [5]. However, for now there is no recognized prognostic tool available for AFP-positive HCC patients after hepatectomy.

Nobuoka et al. found that the negative/positive changes before and after hepatectomy can predict the postoperative prognosis of HCC patients [6]. Another study showed that the prognosis of AFP-positive HCC patients with a postoperative decrease of more than $50 \%$ AFP is better than that of patients with a postoperative decrease of less than $50 \%$ [7]. These studies indicate that the before or after operative change of the AFP level can be used as a predictive indicator of HCC especially AFP-positive HCC. 
TABLE 1: The clinical feature of patients in training cohort and validation cohort.

\begin{tabular}{|c|c|c|c|}
\hline & Training cohort & Validation cohort & $p$ value \\
\hline Number & 439 & 440 & \\
\hline Age (year) & $49.46 \pm 11.61$ & $50.11 \pm 12.45$ & 0.420 \\
\hline \multicolumn{4}{|l|}{ Gender } \\
\hline Male & $374(85.2)$ & $387(88.0)$ & 0.230 \\
\hline Female & $65(14.8)$ & $53(12.0)$ & \\
\hline \multicolumn{4}{|l|}{ Smoking history, $n$ (\%) } \\
\hline Yes & $193(44.0)$ & $203(46.1)$ & 0.792 \\
\hline No & $246(56.0)$ & $237(53.9)$ & \\
\hline \multicolumn{4}{|l|}{ Alcohol history, $n(\%)$} \\
\hline Yes & $93(21.2)$ & $109(24.8)$ & 0.206 \\
\hline No & $346(78.8)$ & $331(75.2)$ & \\
\hline \multicolumn{4}{|l|}{ HBsAg, $n(\%)$} \\
\hline Positive & $380(86.6)$ & $371(84.3)$ & 0.346 \\
\hline Negative & $59(13.4)$ & $69(15.7)$ & \\
\hline \multicolumn{4}{|l|}{ Cirrhosis, $n(\%)$} \\
\hline Yes & $426(97.0)$ & $433(98.4)$ & 0.173 \\
\hline No & $13(3.0)$ & $7(1.6)$ & \\
\hline Child-Pugh score & $5.06 \pm 0.31$ & $5.04 \pm 0.23$ & 0.321 \\
\hline \multicolumn{4}{|l|}{ Child-Pugh class } \\
\hline A & $431(98.2)$ & $437(99.3)$ & 0.128 \\
\hline B & $8(1.8)$ & $3(0.7)$ & \\
\hline MELD score & $4.22 \pm 2.80$ & $4.45 \pm 2.65$ & 0.207 \\
\hline ALBI score & $-2.96 \pm 0.32$ & $-2.93 \pm 0.32$ & 0.221 \\
\hline TNM stage, $n(\%)$ & & & 0.505 \\
\hline IA & $53(12.1)$ & $38(8.6)$ & \\
\hline IB & $149(33.4)$ & $155(35.2)$ & \\
\hline II & $160(36.4)$ & $160(36.4)$ & \\
\hline IIIA & $34(7.7)$ & $36(8.2)$ & \\
\hline IIIB & $43(9.8)$ & $51(11.6)$ & \\
\hline Tumor size $(\mathrm{cm})$ & $5.53 \pm 3.64$ & $5.54 \pm 3.39$ & 0.950 \\
\hline Differentiation, $n(\%)$ & & & 0.869 \\
\hline Well-moderate & $186(42.4)$ & $184(41.8)$ & \\
\hline Poor-undifferentiated & $253(57.6)$ & $256(58.2)$ & \\
\hline Microscopic vascular invasion, $n(\%)$ & & & 0.289 \\
\hline Yes & $182(41.5)$ & $198(45.0)$ & \\
\hline No & $257(58.5)$ & $242(55.0)$ & \\
\hline Macroscopic vascular invasion, $n(\%)$ & & & 0.740 \\
\hline Yes & $41(9.3)$ & $44(10.0)$ & \\
\hline No & $388(90.7)$ & $396(90.0)$ & \\
\hline Tumor multiplicity, $n(\%)$ & & & 0.296 \\
\hline Single & $349(79.5)$ & $362(82.3)$ & \\
\hline Multiple & $90(20.5)$ & $78(17.7)$ & \\
\hline AFP before hepatectomy $(\mathrm{ng} / \mu \mathrm{l})$ & $12889.34 \pm 33411.38$ & $9818.80 \pm 25108.39$ & 0.124 \\
\hline AFP after hepatectomy $(\mathrm{ng} / \mu \mathrm{l})$ & $303.41 \pm 1148.90$ & $275.51 \pm 1458.27$ & 0.753 \\
\hline AFP ratio & $90.97 \pm 212.37$ & $92.68 \pm 160.20$ & 0.892 \\
\hline
\end{tabular}


TABLe 2: The clinical feature of patients with AFP ratio high and AFP ratio low in 2 cohort.

\begin{tabular}{|c|c|c|c|c|c|c|}
\hline & \multicolumn{2}{|c|}{ Training cohort } & \multirow{2}{*}{$p$ value } & \multicolumn{2}{|c|}{ Validation cohort } & \multirow{2}{*}{$p$ value } \\
\hline & AFP ratio high & AFP ratio low & & AFP ratio high & AFP ratio low & \\
\hline Number & 306 & 133 & & 318 & 122 & \\
\hline Age & $49.15 \pm 12.00$ & $50.16 \pm 10.68$ & 0.406 & $50.04 \pm 12.46$ & $50.30 \pm 12.48$ & 0.124 \\
\hline \multicolumn{7}{|l|}{ Gender } \\
\hline Male & $254(83.0)$ & $120(90.2)$ & 0.050 & $275(86.5)$ & $112(91.8)$ & 0.051 \\
\hline Female & $52(17.0)$ & $13(9.8)$ & & $43(13.5)$ & $10(8.2)$ & \\
\hline \multicolumn{7}{|l|}{ Smoking history, $n$ (\%) } \\
\hline Yes & $131(42.8)$ & $62(46.6)$ & 0.460 & $148(46.5)$ & $55(45.1)$ & 0.783 \\
\hline No & $175(57.2)$ & $71(53.4)$ & & $170(53.5)$ & $67(54.9)$ & \\
\hline \multicolumn{7}{|l|}{ Alcohol history, $n(\%)$} \\
\hline Yes & $60(19.6)$ & $33(24.8)$ & 0.220 & $80(25.2)$ & $29(23.8)$ & 0.763 \\
\hline No & $246(80.4)$ & $100(75.2)$ & & $238(74.8)$ & $93(76.2)$ & \\
\hline \multicolumn{7}{|l|}{ HBsAg, $n(\%)$} \\
\hline Positive & $266(86.9)$ & $114(85.7)$ & 0.732 & $262(82.4)$ & $109(89.3)$ & 0.073 \\
\hline Negative & $40(13.1)$ & $19(14.3)$ & & $56(17.6)$ & $13(10.7)$ & \\
\hline \multicolumn{7}{|l|}{ Cirrhosis, $n(\%)$} \\
\hline Yes & $298(97.4)$ & $128(96.2)$ & 0.515 & $316(99.4)$ & $117(95.9)$ & 0.009 \\
\hline No & $8(2.6)$ & $5(3.8)$ & & $2(0.6)$ & $5(4.1)$ & \\
\hline Child-Pugh score & $5.05 \pm 0.26$ & $5.10 \pm 0.39$ & 0.103 & $5.03 \pm 0.19$ & $5.07 \pm 0.32$ & 0.090 \\
\hline \multicolumn{7}{|l|}{ Class } \\
\hline A & $302(98.7)$ & $129(97.0)$ & 0.221 & 317 (99.7) & $120(98.4)$ & 0.131 \\
\hline $\mathrm{B}$ & $4(1.3)$ & $4(3.0)$ & & $1(0.3)$ & $2(1.6)$ & \\
\hline MELD score & $4.11 \pm 2.56$ & $4.47 \pm 3.27$ & 0.214 & $4.26 \pm 2.65$ & $4.96 \pm 2.61$ & 0.013 \\
\hline ALBI score & $-2.99 \pm 0.30$ & $-2.89 \pm 0.34$ & 0.003 & $-2.95 \pm 0.31$ & $-2.89 \pm 0.33$ & 0.056 \\
\hline \multicolumn{7}{|l|}{ TNM stage, $n(\%)$} \\
\hline IA & $40(13.1)$ & $13(9.8)$ & 0.319 & $30(9.4)$ & $8(6.6)$ & 0.334 \\
\hline IB & $102(33.3)$ & $47(35.3)$ & & $118(37.1)$ & $37(30.3)$ & \\
\hline II & $106(34.6)$ & $54(40.6)$ & & $110(34.6)$ & $50(41.0)$ & \\
\hline IIIA & $23(7.5)$ & $11(8.3)$ & & $27(8.5)$ & $9(7.4)$ & \\
\hline IIIB & $35(11.4)$ & $8(6.0)$ & & $33(10.4)$ & $18(14.8)$ & \\
\hline Tumor size $(\mathrm{cm})$ & $5.61 \pm 3.54$ & $5.34 \pm 3.86$ & 0.470 & $5.62 \pm 3.34$ & $5.35 \pm 3.51$ & 0.459 \\
\hline \multicolumn{7}{|l|}{ Differentiation, $n(\%)$} \\
\hline Well-moderate & $173(56.5)$ & $80(60.2)$ & 0.481 & $187(58.8)$ & $69(56.6)$ & 0.669 \\
\hline Poor-undifferentiated & $133(43.5)$ & $53(39.8)$ & & $131(41.2)$ & $53(43.3)$ & \\
\hline \multicolumn{7}{|l|}{ Microscopic vascular invasion, $n(\%)$} \\
\hline Yes & $129(42.2)$ & $53(39.8)$ & 0.652 & $140(44.0)$ & $58(47.5)$ & 0.507 \\
\hline No & $177(57.8)$ & $80(60.2)$ & & $178(56.0)$ & $64(52.5)$ & \\
\hline \multicolumn{7}{|l|}{ Macroscopic vascular invasion, $n(\%)$} \\
\hline Yes & $33(10.8)$ & $8(6.0)$ & 0.115 & $29(9.1)$ & $15(12.3)$ & 0.320 \\
\hline No & $273(89.2)$ & $125(94.0)$ & & $289(90.9)$ & $107(87.7)$ & \\
\hline \multicolumn{7}{|l|}{ Tumor multiplicity, $n(\%)$} \\
\hline Single & $249(81.4)$ & $100(75.2)$ & 0.140 & $264(83.0)$ & $98(80.3)$ & 0.508 \\
\hline Multiple & $57(18.6)$ & $33(24.8)$ & & $54(17.0)$ & $24(19.7)$ & \\
\hline AFP before hepatectomy $(\mathrm{ng} / \mu \mathrm{l})$ & $16564.39 \pm 37311.78$ & $4433.95 \pm 19597.33$ & $<0.001$ & $12099.86 \pm 26777.70$ & $3873.06 \pm 18958.05$ & 0.002 \\
\hline AFP after hepatectomy (ng/ $\mu \mathrm{l})$ & $227.66 \pm 667.27$ & $477.70 \pm 1818.57$ & 0.036 & $181.74 \pm 629.40$ & $519.92 \pm 2567.91$ & 0.029 \\
\hline
\end{tabular}




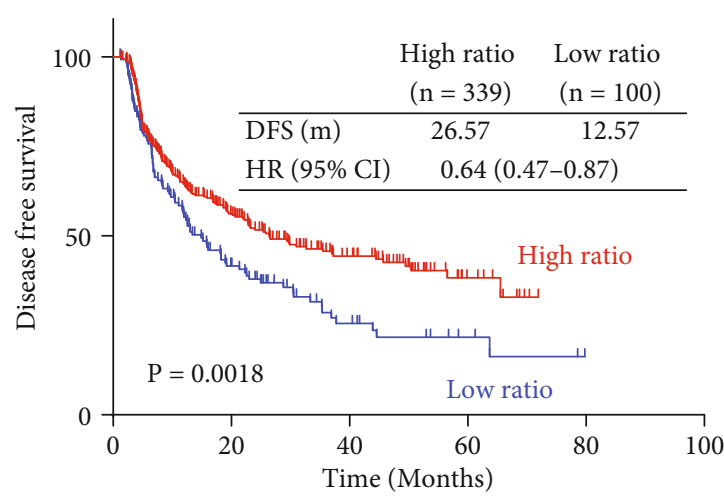

(a)

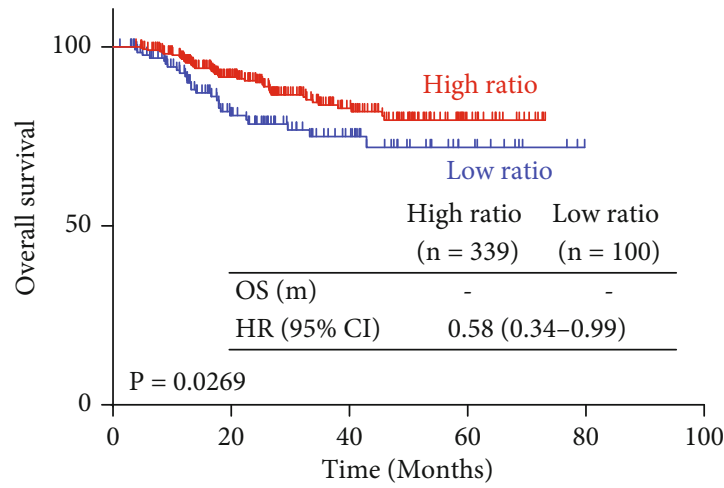

(c)

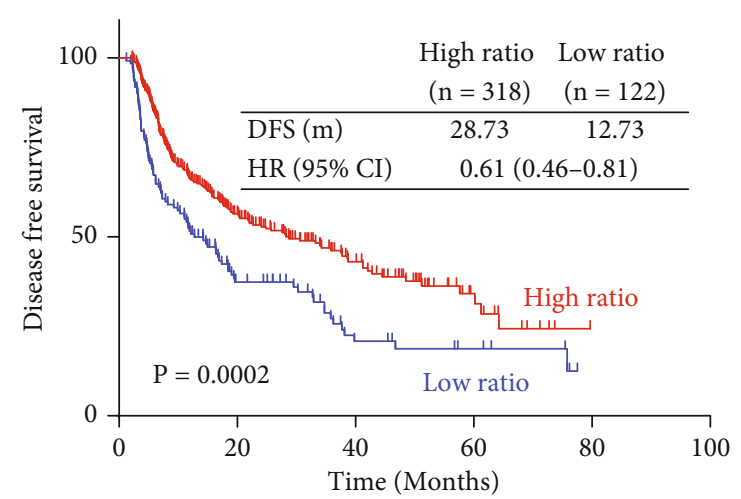

(b)

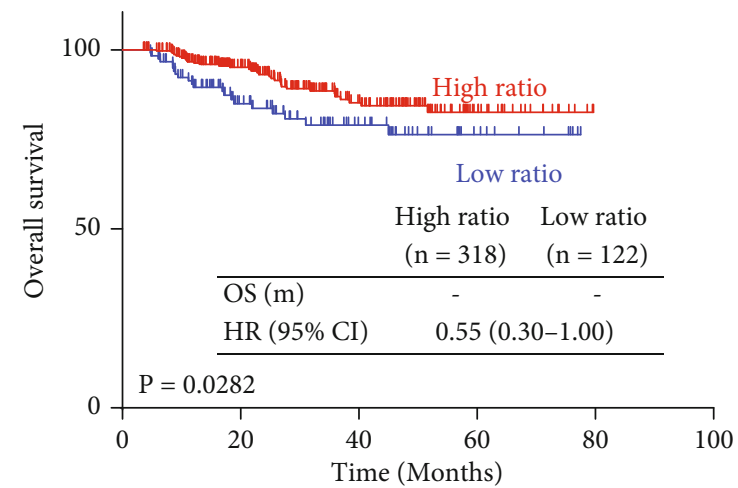

(d)

FIGURE 1: Kaplan-Meier curves showing the DFS of AFP ratio high and AFP ratio low HCC patients with AFP-positive in the training cohort (a) and validation cohort (b). Kaplan-Meier curves showing the OS of AFP ratio high and AFP ratio low HCC patients with AFP-positive in the training cohort (c) and validation cohort (d). AFP: alpha-fetoprotein; HCC: hepatocellular carcinoma; DFS: diseasefree survival; OS: overall survival.

Therefore, in this retrospective study, we aimed at investigating the prognosis effect of AFP level before/after operation ratio (AFP ratio) in AFP-positive HCC patients after surgery.

\section{Materials and Methods}

2.1. Patient Enrollment. From February 2012 to October 2017, a total of 879 HCC patient undergoing resection at Sun Yat-sen University Cancer Center were selected for this retrospective analysis. Inclusion criteria included the following: (1) diagnosed with HCC confirmed by pathological examination; (2) hepatectomy as first-line treatment; (3) R0 resection; (4) preoperative serum AFP level was positive; (5) all patients had a well-documented clinical history and detailed follow-up information. The exclusion criteria included second primary tumor and distant metastasis. All patients were regrouped according to the 8th American Joint Committee on Cancer (AJCC) tumor-node-metastasis (TNM) staging system. Ethics approval was given by the institution ethics committee (approval number B2019-019-01).

2.2. AFP Ratio Calculated. The AFP ratio was defined as the ratio of AFP values within one week before hepatectomy to AFP values within 20-40 days after surgery. The normal range of AFP in the hospital is $0-25 \mathrm{ng} / \mu \mathrm{l}$.
2.3. Follow-Up. This study endpoints were overall survival (OS) and disease-free survival (DFS). The subsequent follow-up after surgery was conducted according to the National Comprehensive Cancer Network guidelines, including serum AFP levels, enhanced computed tomography (CT), or enhanced magnetic resonance imaging (MRI) in every 3 months during the first 2 years after hepatectomy and every 6 months thereafter. Recurrence is according to enhanced CT, MRI, or medical record.

2.4. Statistical Analysis. Statistical analysis was performed using SPSS software (SPSS version 20.0, SPSS). Continuous data were analyzed using the $t$-test and one-way analysis of variance (ANOVA). Categorical data were tested using the chi-square test. The ROC curve was used to evaluate the prognostic accuracy of the AFP ratio and AFP value before hepatectomy. Survival curves were estimated by the Kaplan-Meier (KM) method. A 2-tail $p$ value below 0.05 are considered significant.

\section{Results}

3.1. Clinical Characteristics of AFP-Positive HCC Patients. The median follow-up time was 25.57 months. Patients were grouped into a training cohort $(n=439)$ and a validation cohort $(n=440)$ with random number method [8]. The 

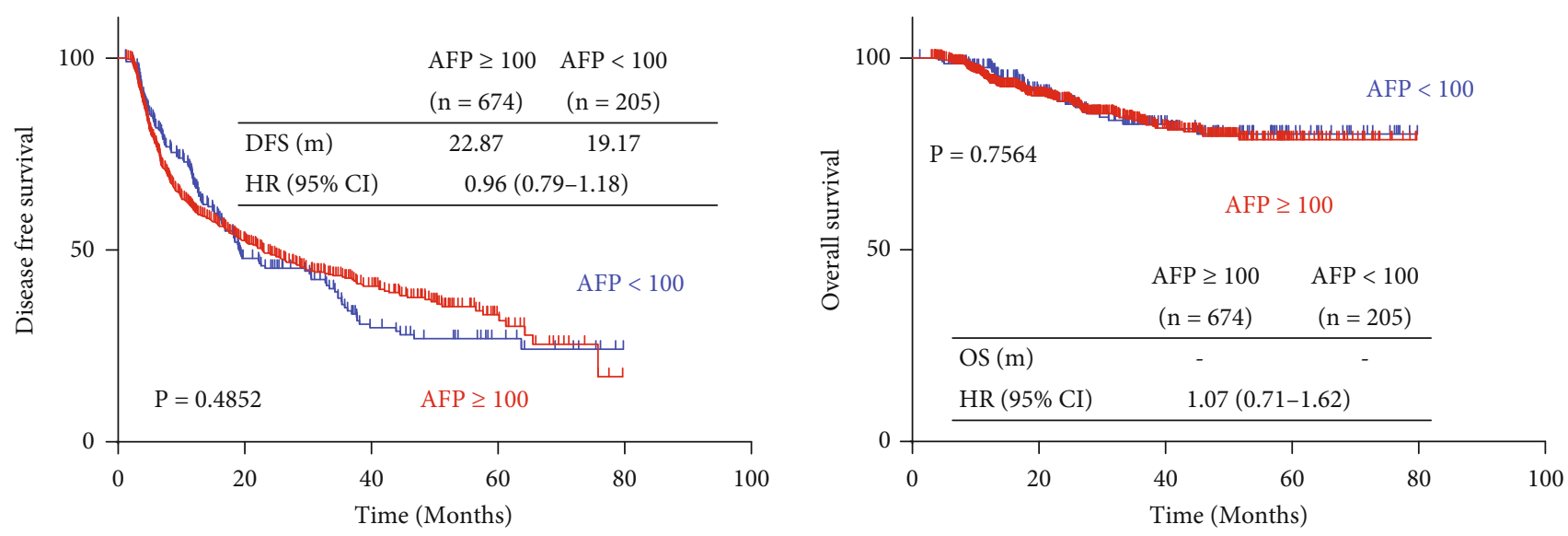

(a)
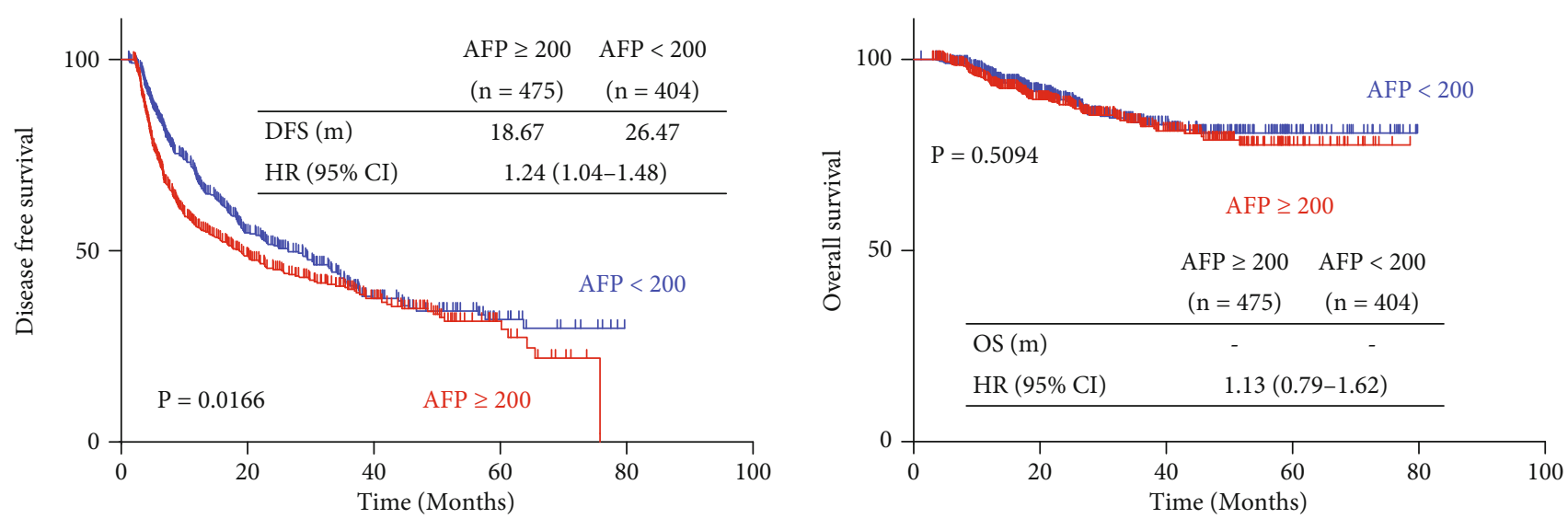

(b)
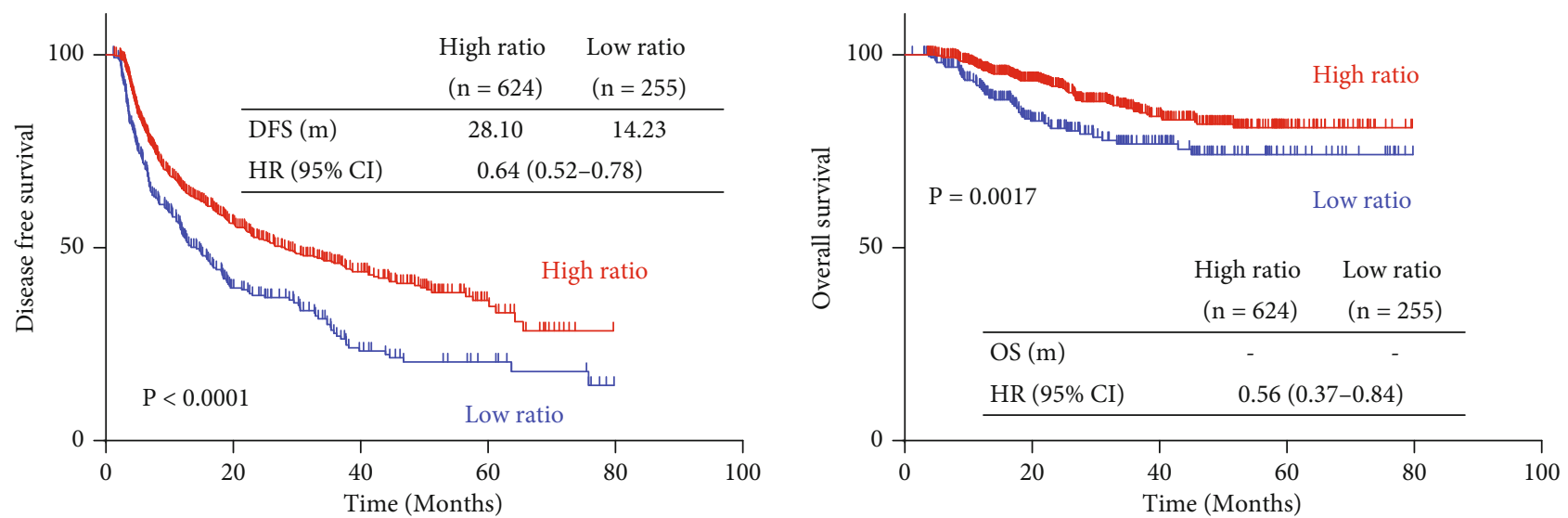

(c)

FIGURE 2: Kaplan-Meier curves showing the DFS and OS of preoperation AFP level $\geq 100 \mathrm{ng} / \mu \mathrm{l}$ (a), preoperation AFP level $\geq 200 \mathrm{ng} / \mu \mathrm{l}$ (b), and AFP ratio (c) in AFP-positive HCC patients. AFP: alpha-fetoprotein; HCC: hepatocellular carcinoma; DFS: disease-free survival; OS: overall survival.

results showed that there were no statistically significant differences in the clinical characteristics between the two cohorts (see Table 1).

3.2. Association between AFP Ratio and Clinical Characteristics of AFP-Positive HCC Patients. We used Xtile software to determine the AFP ratio cut-off value (17.8) in training cohort [9]. In the training cohort, the ALBI score of patients with AFP ratio $>17.8$ (AFP ratio high) was lower than patients with AFP ratio $\leq 17.8$ (AFP ratio low) (AFP ratio high vs. AFP ratio low, $-2.99 \pm 0.30$ vs. -2.89 $\pm 0.34, p=0.003)$. In the validation cohort, the patients with or without cirrhosis had a significant difference between AFP ratio high and AFP ratio low $(p=0.009)$. The AFP before hepatectomy of AFP ratio high was higher than AFP ratio low and the AFP after hepatectomy of AFP ratio 


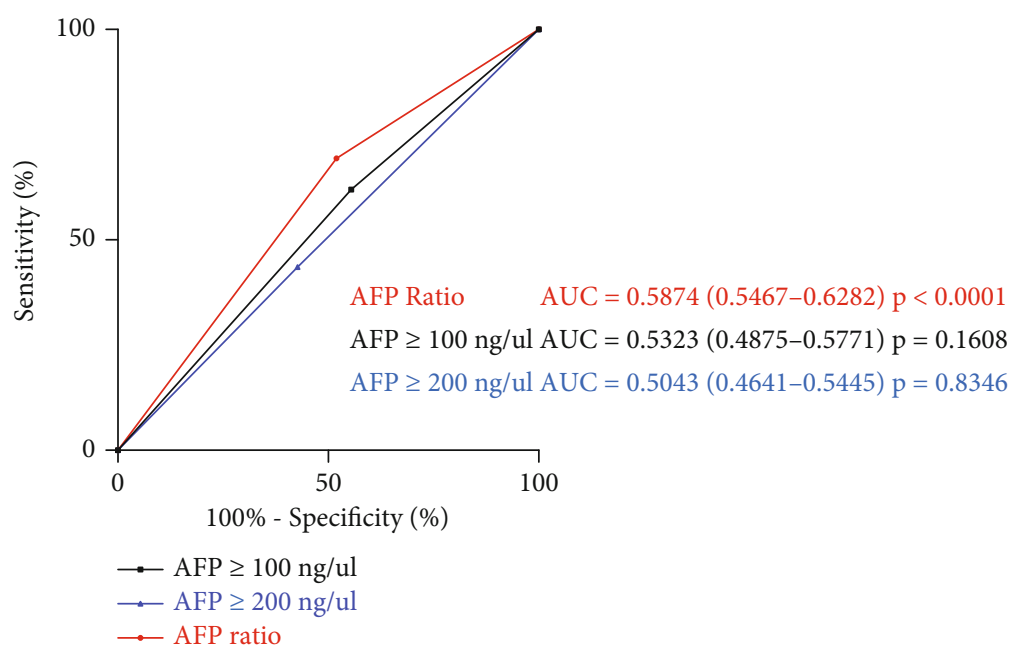

(a)

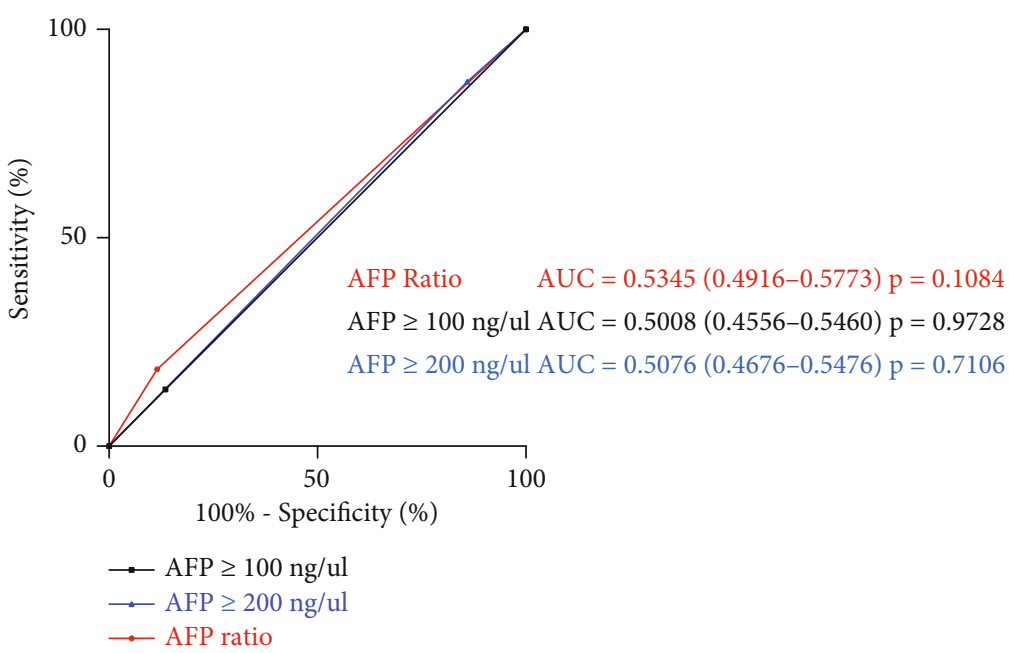

(b)

Figure 3: Comparison of ROC curves of preoperation AFP level $\geq 100 \mathrm{ng} / \mu \mathrm{l}$, preoperation AFP level $\geq 200 \mathrm{ng} / \mu \mathrm{l}$, and AFP ratio in prognostic evaluation of DFS (a) and OS (b) in AFP-positive HCC patients. AFP: alpha-fetoprotein; HCC: hepatocellular carcinoma; DFS: disease-free survival; OS: overall survival.

high was lower than AFP ratio low, as we had expected. The other clinical and laboratory parameters did not vary between AFP ratio high and AFP ratio low in the two cohorts (see Table 2).

3.3. Prognostic Value of AFP Ratio in Patients with AFPPositive HCC. We next investigated the prognostic value of AFP ratio in AFP-positive HCC patients after resection. In the training cohort, the DFS (26.57 months with AFP ratio high vs. 12.57 months with AFP ratio low, $p=0.0018$; HR, $0.64,95 \% \mathrm{CI}, 0.47-0.87)$ and OS ( $p=0.0269$; HR, 0.58, 95\% CI, 0.34-0.99) of AFP ratio high patients was significantly longer than AFP ratio low patients. In the validation cohort, the DFS (28.73 months with AFP ratio high vs. 12.73 months with AFP ratio low, $p=0.0020$; HR, 0.61, 95\% CI, 0.46-0.81) and OS ( $p=0.0282$; HR, $0.55,95 \% \mathrm{CI}, 0.30-1.00)$ of AFP ratio high patients was significantly longer than for those that were AFP ratio low patients (see Figure 1).
We further analyzed the predictive value of AFP ratio in early HCC recurrence and later HCC recurrence in the whole patients. According to previous study, early recurrence was defined as recurrence within 1 year after surgery and later recurrence was defined as recurrence after 1 year [10]. The results showed no statistically significant difference of AFP ratio between early HCC recurrence patients and later HCC recurrence patients (early recurrence vs. later recurrence, $78.06 \pm 8.23$ vs. $90.78 \pm 14.42, p=0.41$ ) (see Supplementary Figure (available here)). The proportion of AFP ratio high among early recurrence was similar to that among later recurrence (AFP ratio high in early recurrence was $64.55 \%$ (213/330); AFP ratio high in later recurrence was $64.91 \%(111 / 171))$.

3.4. AFP Level in the Prognostic Value of HCC Patients. $\mathrm{AFP} \geq 100 \mathrm{ng} / \mu \mathrm{l}$ or AFP $\geq 200 \mathrm{ng} / \mu \mathrm{l}$ is generally considered to be a useful indicator for HCC patients prognostic after 
TABLE 3: Univariate and multivariate analyses of the disease-free survival of AFP-positive HCC patients.

\begin{tabular}{|c|c|c|c|c|}
\hline & \multicolumn{2}{|c|}{ Univariate } & \multicolumn{2}{|c|}{ Multivariate } \\
\hline & HR (95\% CI) & $p$ value & $\mathrm{HR}(95 \% \mathrm{CI})$ & $p$ value \\
\hline Gender (male) & $1.467(1.100-1.955)$ & 0.009 & $1.231(0.921-1.646)$ & 0.160 \\
\hline Age $<50$ years & $1.168(0.980-1.392)$ & 0.083 & & \\
\hline Smoking & $1.134(0.951-1.352)$ & 0.161 & & \\
\hline Drinking & $1.216(0.994-1.487)$ & 0.057 & & \\
\hline HBV infected & $0.885(0.692-1.131)$ & 0.330 & & \\
\hline Cirrhosis & $1.146(0.646-2.032)$ & 0.641 & & \\
\hline Child-Pugh A & $0.777(0.368-1.639)$ & 0.508 & & \\
\hline TNM stage III & $2.397(1.959-2.933)$ & $<0.001$ & $0.779(0.549-1.105)$ & 0.162 \\
\hline Tumor size $>5 \mathrm{~cm}$ & $1.885(1.581-2.247)$ & $<0.001$ & $0.697(0.553-0.878)$ & 0.002 \\
\hline Tumor size $>3 \mathrm{~cm}$ & $1.785(1.458-2.185)$ & $<0.001$ & $0.828(0.644-1.065)$ & 0.141 \\
\hline Differentiation poor & $1.229(1.027-1.469)$ & 0.024 & $1.110(0.927-1.330)$ & 0.257 \\
\hline Microscopic vascular invasion & $1.876(1.573-2.238)$ & $<0.001$ & $1.472(1.220-1.776)$ & $<0.001$ \\
\hline Macroscopic vascular invasion & $2.316(1.788-3.000)$ & $<0.001$ & $1.537(1.055-2.240)$ & 0.025 \\
\hline Tumor multiplicity & $1.730(1.411-2.121)$ & $<0.001$ & $1.325(1.029-1.706)$ & 0.029 \\
\hline $\mathrm{AFP}>100 \mathrm{ng} / \mu \mathrm{l}$ & $0.964(0.788-1.180)$ & 0.725 & & \\
\hline $\mathrm{AFP}>200 \mathrm{ng} / \mu \mathrm{l}$ & $1.104(0.918-1.328)$ & 0.295 & & \\
\hline AFP ratio $<17.8$ & $1.572(1.308-1.888)$ & $<0.001$ & $1.710(1.422-2.056)$ & $<0.001$ \\
\hline
\end{tabular}

surgery $[11,12]$. We compared AFP ratio and AFP level in predicting prognosis of AFP-positive HCC patients after surgery. The results showed that no difference in DFS or OS was found between AFP $\geq 100 \mathrm{ng} / \mu \mathrm{l}$ patients and AFP $<100 \mathrm{ng} / \mu \mathrm{l}$ patients (DFS, AFP $\geq 100 \mathrm{ng} / \mu \mathrm{l}$ vs. AFP $<100$ $\mathrm{ng} / \mu \mathrm{l}, 22.87$ vs. 19.17 months, $p=0.4852$; HR, $0.96,95 \%$ CI, 0.79-1.18; OS, $p=0.7564$; HR, 1.07, 95\% CI, 0.71-1.62). $\mathrm{AFP} \geq 200 \mathrm{ng} / \mu \mathrm{l}$ can predict the DFS of AFP-positive HCC patients after hepatectomy (AFP $\geq 200 \mathrm{ng} / \mu \mathrm{l}$ vs. AFP $<200$ $\mathrm{ng} / \mu \mathrm{l}, 18.67$ vs. 26.47 months, $p=0.0166$; HR, $1.24,95 \%$ CI, 1.04-1.48), but not OS ( $p=0.5094$; HR, $1.13,95 \% \mathrm{CI}$, 0.79-1.62). The DFS of HCC patients with high AFP ratio was significantly longer than that of patients with low AFP ratio (AFP ratio high vs. AFP ratio low, 28.10 vs. 14.23 months, $p<0.0001$; HR, 0.64, 95\% CI, 0.52-0.78), and OS was also significantly prolonged $(p=0.0017$; HR, $0.56,95 \%$ CI, 0.37-0.84) (Figure 2).

We used ROC curve to compare the prognostic efficacy of $\mathrm{AFP}$ ratio, $\mathrm{AFP} \geq 100 \mathrm{ng} / \mu \mathrm{l}$, and $\mathrm{AFP} \geq 200 \mathrm{ng} / \mu \mathrm{l}$. With an AUC of 0.5875 (95\% CI, 0.5467-0.6282) on prediction of recurrence, AFP ratio outperformed AFP level (AFP $\geq 100 \mathrm{ng} / \mu \mathrm{l}$, $\mathrm{AUC}=0.5323 \quad(95 \% \mathrm{CI}, 0.4875-0.5771) ; \mathrm{AFP} \geq 200 \mathrm{ng} / \mu \mathrm{l}$, $\mathrm{AUC}=0.5043$ (95\% CI, 0.4641-0.5445)). In OS prediction, the AUC of the AFP ratio was 0.5345 (95\% CI, 0.49160.5773 ), which was similar to AFP level (AFP $\geq 100 \mathrm{ng} / \mu \mathrm{l}$, $\mathrm{AUC}=0.5008 \quad(95 \%$ CI, 0.4556-0.5460); $\mathrm{AFP} \geq 200 \mathrm{ng} / \mu \mathrm{l}$, $\mathrm{AUC}=0.5076$ (95\% CI, 0.4676-0.5476)) (Figure 3).

3.5. Univariate and Multivariate Survival Analyses in Patients with AFP-Positive HCC. The results of the univariate and multivariate analysis were listed in Tables 3 and 4. Multivariate analysis indicated that AFP ratio $>17.8$ was an independent predictor of DFS and OS of AFP-positive
HCC patients (DFS, HR, 1.710, 95\% CI, 1.422-2.056, $p<$ 0.001 ; OS, HR, 2.004, 95\% CI, 1.381-2.906, $p<0.001)$. The results showed that tumor size $>5 \mathrm{~cm}$, microscopic vascular invasion, macroscopic vascular invasion, and tumor multiplicity were also independent poor prognostic factors for AFP-positive HCC patients regarding DFS but not OS (Tables 3 and 4).

\section{Discussion}

Clinically, AFP-positive HCC patients presented high malignancy, rapid progression and poor prognosis compared to AFP-negative HCC patients [13]. Previous research showed AFP-positive HCC patients with 5-year survival rate of 26.7\% compared to AFP-negative HCC patients with 5year survival rate of $56.5 \%$ [14]. However, there was lack of efficient prognostic indicators of AFP-positive HCC patients after surgery. In this study, we explored the role of AFP ratio in AFP-positive HCC patients after surgery by conducting a retrospective analysis.

AFP is wildly used for diagnosis, prognosis, and surveillance of HCC [15]. According to the level of serum AFP that can be used clinically, we generally divide the HCC patients to AFP-positive and AFP-negative [16]. HCC patients with AFP-positive and AFP-negative showed significant differences in clinical. Prior studies have suggested the association between high AFP levels and poor outcome of patients with HCC and some of staging systems like the Cancer of the Liver Italian Pro-gram score (CLIP score) [17] and Biomarker combined Japan Integrated Staging (bm-JIS) [18] already incorporated the level of serum AFP [19]. Therefore, only few studies focused on prognostic biomarkers in AFP- 
TABLE 4: Univariate and multivariate analyses of the overall survival of AFP-positive HCC patients.

\begin{tabular}{|c|c|c|c|c|}
\hline & \multicolumn{2}{|l|}{ Univariate } & \multicolumn{2}{|c|}{ Multivariate } \\
\hline & $\operatorname{HR}(95 \% \mathrm{CI})$ & $p$ value & HR $(95 \% \mathrm{CI})$ & $p$ value \\
\hline Gender (male) & $1.343(0.740-2.439)$ & 0.332 & & \\
\hline Age $<50$ year & $0.976(0.681-1.398)$ & 0.894 & & \\
\hline Smoking & $1.134(0.951-1.352)$ & 0.654 & & \\
\hline Drinking & $1.655(1.131-2.421)$ & 0.009 & $1.467(0.998-2.155)$ & 0.051 \\
\hline HBV infected & $0.896(0.543-1.480)$ & 0.668 & & \\
\hline Cirrhosis & $0.817(0.302-2.216)$ & 0.692 & & \\
\hline Child-Pugh A & $20.419(0.005-75989.228)$ & 0.472 & & \\
\hline TNM stage III & $3.015(2.072-4.389)$ & $<0.001$ & $0.610(0.309-1.206)$ & 0.155 \\
\hline Tumor size $>5 \mathrm{~cm}$ & $2.381(1.648-3.439)$ & $<0.001$ & $0.679(0.418-1.101$ & 0.116 \\
\hline Tumor size $>3 \mathrm{~cm}$ & $2.492(1.554-3.994)$ & $<0.001$ & $0.670(0.375-1.196)$ & 0.176 \\
\hline Differentiation poor & $1.489(1.024-2.166)$ & 0.037 & $0.667(0.417-1.099)$ & 0.114 \\
\hline Microscopic vascular invasion & $1.952(1.359-2.805)$ & $<0.001$ & $1.334(0.902-1.974)$ & 0.148 \\
\hline Macroscopic vascular invasion & $2.699(1.697-4.294)$ & $<0.001$ & $0.598(0.306-1.169)$ & 0.133 \\
\hline Tumor multiplicity & $1.771(1.185-2.647)$ & 0.005 & $1.251(0.738-2.120)$ & 0.407 \\
\hline $\mathrm{AFP}>100 \mathrm{ng} / \mu \mathrm{l}$ & $1.069(0.700-1.634)$ & 0.756 & & \\
\hline $\mathrm{AFP}>200 \mathrm{ng} / \mu \mathrm{l}$ & $1.176(0.800-1.729)$ & 0.410 & & \\
\hline AFP ratio $<17.8$ & $1.785(1.236-2.578)$ & 0.002 & $2.004(1.381-2.906)$ & $<0.001$ \\
\hline
\end{tabular}

positive HCC; others mainly focused on AFP-negative HCC or overall HCC patients.

Toro et al. found AFP level of pre- and posttreatment correlated with survival of HCC patients [20]. Nobuoka et al. also showed that the positive and negative changes of AFP before and after hepatectomy can also predict the postoperative recurrence of HCC [6]. Another study showed that AFP ratio could predict recurrence in HCC after liver transplant [21]. In the present study, we randomly separated AFP-positive $\mathrm{HCC}$ patients into a training cohort and validation cohort and found that HCC patients with high AFP ratio had better OS and DFS in the two cohorts. The results suggested that AFP ratio could be a potential prognostic biomarker in AFP-positive HCC patients after hepatectomy.

Multiple studies have indicated that AFP level could be used as a prognostic marker of HCC patients after hepatectomy [11, 12]. However, the effect of AFP level on AFP-positive HCC after resection is still unclear. Our study found that preoperative $\mathrm{AFP} \geq 100 \mathrm{ng} / \mu \mathrm{l}$ could not predict the prognosis of AFP-positive HCC patients. Preoperative AFP $\geq 200 \mathrm{ng} / \mu \mathrm{l}$ was only related to DFS, but not OS. ROC curve also showed that AFP ratio had better prognostic diagnostic efficacy for HCC patients with AFPpositive than AFP $\geq 100 \mathrm{ng} / \mu \mathrm{l}$ and $\mathrm{AFP} \geq 200 \mathrm{ng} / \mu \mathrm{l}$. The above results suggested a limited prognostic role of AFP level before hepatectomy and AFP ratio could be used as a better prognostic indicator for AFP-positive HCC patients.

Univariate and multivariate analysis also showed that AFP ratio was an independent risk predictor in DFS and
OS in AFP-positive HCC patients. Previous studies have identified several prognostic indicators for HCC patients after hepatectomy, including tumor size [22], TNM stage [22], and vascular invasion [23]. However, these indicators were found no significant difference in OS in this study. Therefore, AFP ratio is a prognosis predictor of $\mathrm{HCC}$ patients with AFP-positive.

Our study showed AFP ratio as a prognostic marker in AFP-positive HCC after surgery. However, there were several shortcomings in this study: (1) there might be a risk of bias in the study due to one single center retrospective study; (2) the AFP level was quantified by different approaches, and the AFP ratio used might need more validation.

\section{Conclusions}

This study retrospectively analyzed AFP-positive HCC patients undergoing hepatectomy in a large cohort. The AFP ratio showed the prognostic significance of AFPpositive HCC patients after surgery, which was validated in both training and validation sets. AFP ratio showed better prognostic predictive value than AFP levels in AFPpositive HCC patients. This study provided a potential prognostic indicator for AFP-positive HCC patients.

\section{Data Availability}

The data used to support the findings of this study are available from the corresponding author upon request. 


\section{Conflicts of Interest}

The authors declare that there is no conflict of interest regarding the publication of this paper.

\section{Authors' Contributions}

Li-Yue Sun and Wen-Jian Cen contributed equally to this work.

\section{Acknowledgments}

This research supported by the 100 top talents program of Sun Yat-sen University (PT19050301) and Doctoral Workstation Foundation of Guangdong Second Provincial General Hospital (2021BSGZ018).

\section{Supplementary Materials}

Supplementary Figure: Comparison of AFP ratio in AFPpositive HCC patients with early recurrence and later recurrence. (Supplementary Materials)

\section{References}

[1] M. Zhou, H. Wang, X. Zeng et al., "Mortality, morbidity, and risk factors in China and its provinces, 1990-2017: a systematic analysis for the Global Burden of Disease Study 2017," Lancet, vol. 394, no. 10204, pp. 1145-1158, 2019.

[2] N. Umezaki, S. Nakagawa, Y. I. Yamashita et al., "Lysyl oxidase induces epithelial-mesenchymal transition and predicts intrahepatic metastasis of hepatocellular carcinoma," Cancer Science, vol. 110, no. 6, pp. 2033-2043, 2019.

[3] F. Kong, M. Jin, D. Cao, Z. Jia, Y. Liu, and J. Jiang, "Galectin-3 not Galectin-9 as a candidate prognosis marker for hepatocellular carcinoma," PeerJ, vol. 8, article e9949, 2020.

[4] G. Wang, X. Lu, Q. du et al., "Diagnostic value of the $\gamma$-glutamyltransferase and alanine transaminase ratio, alpha-fetoprotein, and protein induced by vitamin $\mathrm{K}$ absence or antagonist II in hepatitis B virus-related hepatocellular carcinoma," Scientific Reports, vol. 10, no. 1, p. 13519, 2020.

[5] T. Zhao, L. Jia, J. Li et al., "Heterogeneities of site-specific Nglycosylation in HCC tumors with low and high AFP con-centrations," Frontiers in Oncology, vol. 10, p. 496, 2020.

[6] D. Nobuoka, Y. Kato, N. Gotohda et al., "Postoperative serum alpha-fetoprotein level is a useful predictor of recurrence after hepatectomy for hepatocellular carcinoma," Oncology Reports, vol. 24, no. 2, pp. 521-528, 2010.

[7] N. Rungsakulkij, W. Suragul, S. Mingphruedhi, P. Tangtawee, P. Muangkaew, and S. Aeesoa, "Prognostic role of alphafetoprotein response after hepatocellular carcinoma resection," World Journal of Clinical Cases, vol. 6, no. 6, pp. 110120, 2018.

[8] A. Sui, X. Chen, J. Shen et al., "Inhibiting the NLRP3 inflammasome with MCC950 ameliorates retinal neovascularization and leakage by reversing the IL- $1 \beta /$ IL- 18 activation pattern in an oxygen-induced ischemic retinopathy mouse model," Cell Death \& Disease, vol. 11, no. 10, p. 901, 2020.

[9] R. L. Camp, M. Dolled-Filhart, and D. L. Rimm, "X-Tile: a new bio-informatics tool for biomarker assessment and out-come- based cut-point optimization," Clinical Cancer Research, vol. 10, no. 21, pp. 7252-7259, 2004.

[10] L. Y. Sun, Y. He, Q. Liu, and F. Wang, "Effect of delta $\alpha$-fetoprotein on the detection of liver cancer recurrence," Translational Cancer Research, vol. 9, no. 10, pp. 6263-6274, 2020.

[11] S. Y. Peng, W. J. Chen, P. L. Lai, Y. M. Jeng, J. C. Sheu, and H. C. Hsu, "High $\alpha$-fetoprotein level correlates with high stage, early recurrence and poor prognosis of hepatocellular carcinoma: significance of hepatitis virus infection, age, p53 and $\beta$-catenin mutations," International Journal of Cancer, vol. 112, no. 1, pp. 44-50, 2004.

[12] A. Hiraoka, K. Michitaka, T. Kumada et al., "Prediction of prognosis of intermediate-stage HCC patients: validation of the tumor marker score in a nationwide database in Japan," Liver Cancer, vol. 8, no. 5, pp. 403-411, 2019.

[13] T. Chen, X. Dai, J. Dai et al., “AFP promotes HCC progression by suppressing the HuR-mediated Fas/FADD apoptotic pathway," Cell Death \& Disease, vol. 11, no. 10, p. 822, 2020.

[14] R. Izumi, K. Shimizu, M. Kiriyama et al., "Alpha-fetoprotein production by hepatocellular carcinoma is prognostic of poor patient survival," Journal of Surgical Oncology, vol. 49, no. 3, pp. 151-155, 1992.

[15] J. D. Debes, P. A. Romagnoli, J. Prieto et al., "Serum biomarkers for the prediction of hepatocellular carcinoma," Cancers (Basel), vol. 13, no. 7, p. 1681, 2021.

[16] J. Sun, Y. Zhao, L. Qin et al., "Metabolomic profiles for HBV related hepatocellular carcinoma including AlphaFetoproteins positive and negative subtypes," Frontiers in Oncology, vol. 9, p. 1069, 2019.

[17] The Cancer of the Liver Italian Program (Clip) Investigators, "A new prognostic system for hepatocellular carcinoma: a retrospective study of 435 patients: the Cancer of the Liver Italian Program (CLIP) investigators," Hepatology, vol. 28, no. 3, pp. 751-755, 1998.

[18] S. Kitai, M. Kudo, Y. Minami et al., "A new prognostic staging system for hepatocellular carcinoma: value of the biomarker combined Japan integrated staging score," Intervirology, vol. 51, Supplement 1, pp. 86-94, 2008.

[19] M. Y. Chan, W. H. She, W. C. Dai et al., "Prognostic value of preoperative alpha-fetoprotein (AFP) level in patients receiving curative hepatectomy- an analysis of 1, 182 patients in Hong Kong," Translational Gastroenterology and Hepatology, vol. 4, p. 52, 2019.

[20] A. Toro, A. Ardiri, M. Mannino et al., "Effect of pre- and posttreatment $\alpha$-fetoprotein levels and tumor size on survival of patients with hepatocellular carcinoma treated by resection, transarterial chemoembolization or radiofrequency ablation: a ret-rospective study," BMC Surgery, vol. 14, no. 1, p. 40, 2014.

[21] C. Koch, T. Bette, O. Waidmann et al., "AFP ratio predicts HCC recurrence after liver transplantation," PLoS One, vol. 15, no. 7, article e0235576, 2020.

[22] Y. Zhang, S. W. Chen, L. L. Liu, X. Yang, S. H. Cai, and J. P. Yun, "A model combining TNM stage and tumor size shows utility in predicting recurrence among patients with hepatocellular carcinoma after resection," Cancer Management and Research, vol. 10, pp. 3707-3715, 2018.

[23] Y. P. Fu, Y. Yi, J. L. Huang et al., "Prognostic nomograms stratify survival of patients with hepatocellular carcinoma without portal vein tumor thrombosis after curative resection," The Oncologist, vol. 22, no. 5, pp. 561-569, 2017. 